\title{
Translation and initial validation of the Persian version of Geriatric Fear of Falling Measure (GFFM)
}

\author{
Negar Sadeghi ${ }^{1}$, Majideh Heravi Karimooi ${ }^{2 *}$ Nahid Rejeh $^{2}$, Ali Montazeri ${ }^{3}$ \\ 1. Shahed University, Faculty of Nursing \&Midwifery, Tehran, Iran \\ 2. Elderly Care Research Center, Faculty of Nursing\& Midwifery, Shahed University, Tehran, Iran \\ 3. Health Metrics Research Center, Iranian Institute for Health Sciences Research, ACECR, Tehran, Iran
}

Received: 8 May 2021

Accepted for publication: 15 May 2021

[EPub a head of print-17 May 2021]

Payesh: $2021 ; 20$ (3): 347-356

\begin{abstract}
Objective (s): Fear of falling is the main fear among older adults and is an independent risk factor for decreased mobility and quality of life. Therefore, a standard tool is needed to assess the fear of falling. The Geriatric Fear of Falling Measure is one of the common tools that used for this purpose. The present study aimed to translate and initially validate the Persian version of the questionnaire in Iran.

Methods: This was a methodological study and was conducted on 200 elderly people. After translating and obtaining opinions of experts, the content and face validity of the questionnaire were determined qualitatively. Then, the validity of the GFFM was assessed through convergent validity (correlation test between the Persian version of the GFFM and the Activities Specific Balance Confidence Scale-ABC questionnaire) and known group comparison. Reliability was evaluated using internal consistency (Cronbach's alpha).

Results: The results obtained from known groups comparison indicated that the questionnaire well differentiated among participants who were differed in self-reported health condition. The convergent validity showed a significant correlation between the Persian version of the GFFM and the ABC questionnaires. The Cronbach's alpha coefficient for the dimensions and the total questionnaire was between 0.75 and 0.89 .

Conclusion: The findings indicated that the Persian version of the GFFM is a valid instrument and can be used in a variety of situations, including clinical and research environments. It is suggested that the future studies assess the structural validity of the GFFM using factor analysis with a bigger sample size.
\end{abstract}

Key Words: Validation, older, Geriatric fear of falling measure

\footnotetext{
* Corresponding author: Shahed University, Opposite Holy Shrine of Imam Khomeini, Khalij Fars Expressway, Tehran, Iran E-mail: heravi@shahed.ac.ir
} 


\title{
ترجمه و اعتباريابى اوليه نسخه فارسى يرسشنامه ترس از افتادن در سالمندان (GFFM)
}

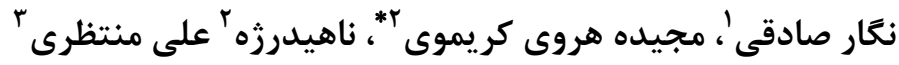

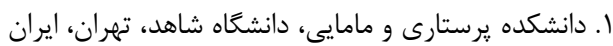

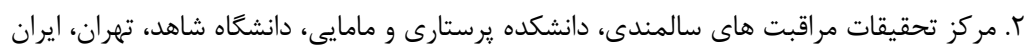

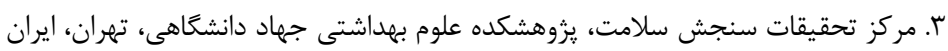

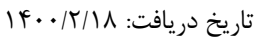

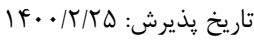

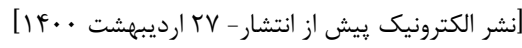 \\ شريه
}

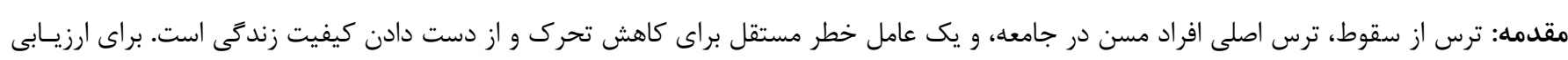

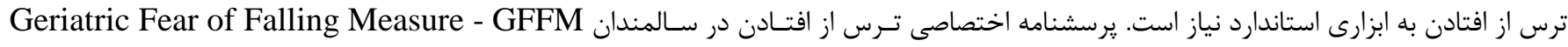

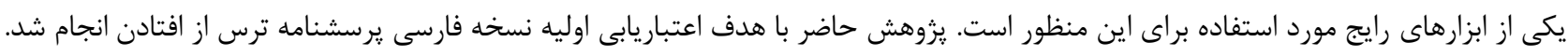

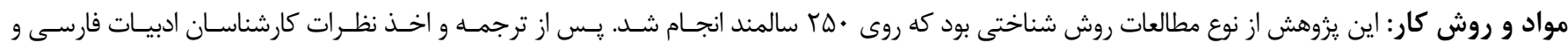

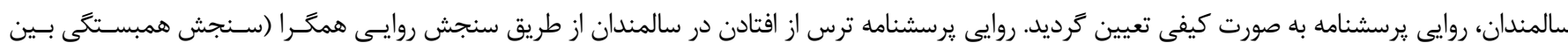

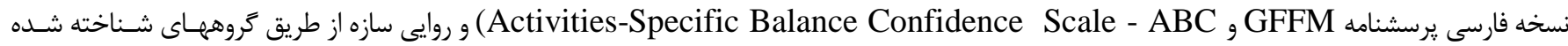

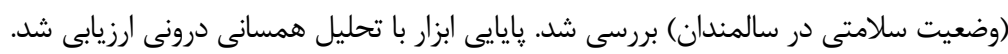

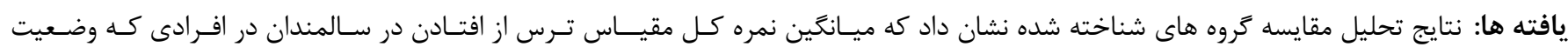

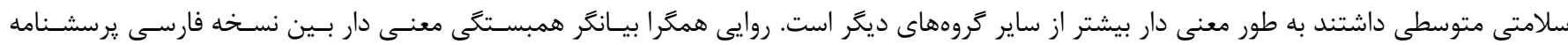

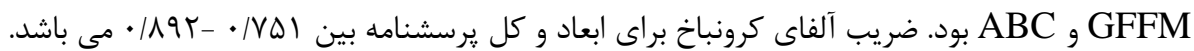

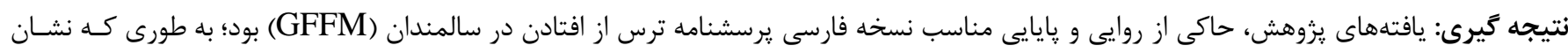

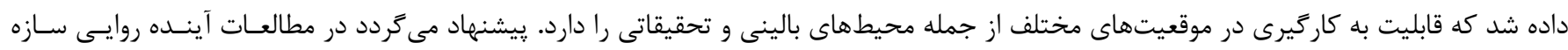

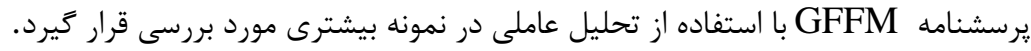

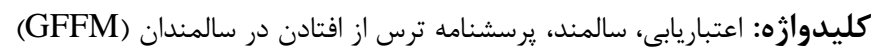




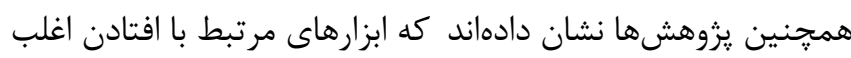

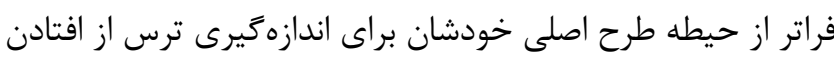

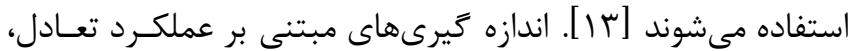

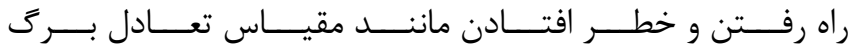
[ [ [ (Berg Balance Scale -BBS)

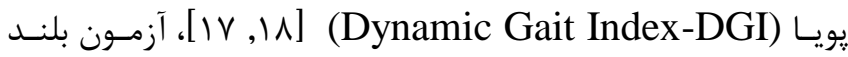

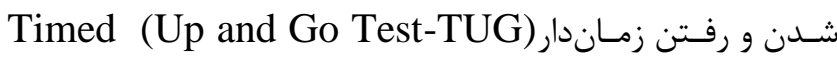
[19]، آزمـون رسـيدن عملكـردى-Functional Reach Test) (r. [FRT)

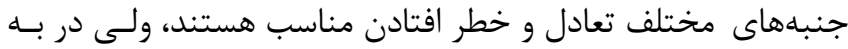
دست آوردن نقش و اثرى كه ترس از افتادن بر فعاليت و شركت در در

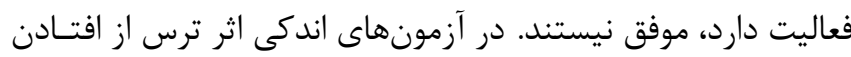

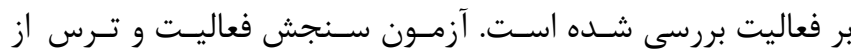
افتـادن در سـالمندان Survey of Activities and Fear of فصال Falling in the Elderly- SAFFE) فعاليتشان را به خاطر ترس از افتادن محدود مى كنند و آنهايى كـهـ النه ترس از افتادن دارند، ولى فعاليتشان را محدود نمى كنند، از هم جدا

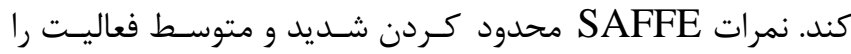

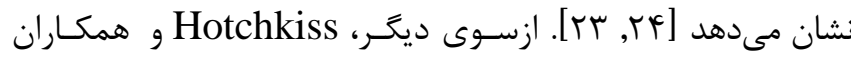
دريافتند SAFFE در ويشبينى دقيـق تكـرار افتـادن، محسدوديت

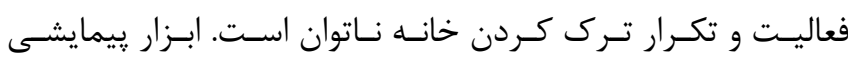

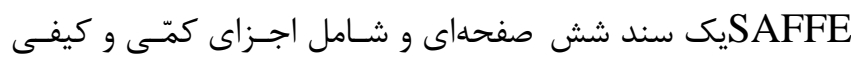

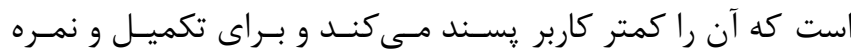

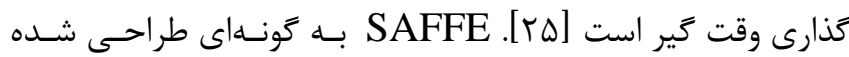

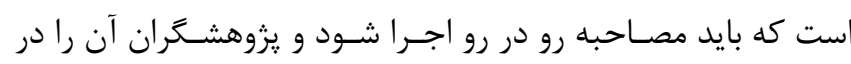

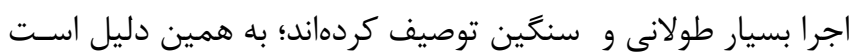

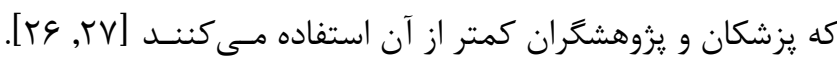
نسخه اصلاح شده سنجش فعاليت و ترس از افتـادن در سـالمندان (Modified Survey of Activities and Fear of IV يـك مقيـاس Falling in the Elderly- mSAFFE) كويه اى در راستاى فعاليتهاى اجتناب شده است ـ ايـن يرسشـنامه به كونهاى طراحى شده است تا يك برسشنامه قابل اجرا توسط فرد

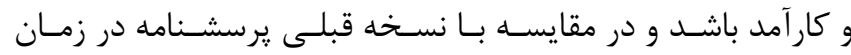
كمترى اجرا، كامل و امتياز كذارى شود [rA]. مطالعـات SAFFE mS را مقايسه و ززارش كردند كه mSAFFE احتمالا
رسيدن به سن سالمندى را بايسـ يكـى از عمــده تـرين ييشـرفتهاى

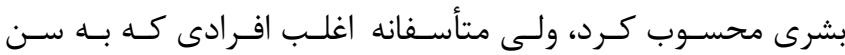

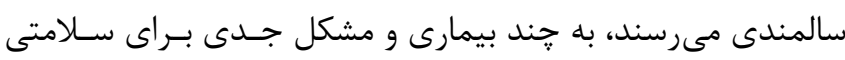

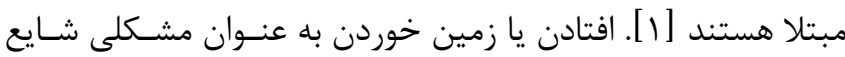

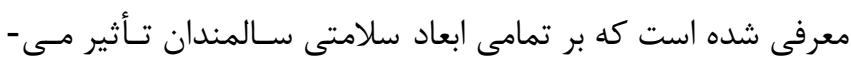

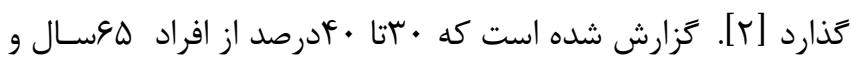
بيشتر، در طول زمان يك سال تجربه افتادن را دارند كه اين امر آنها

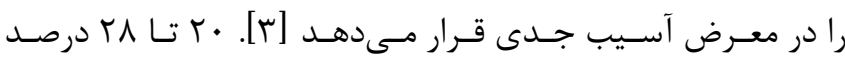

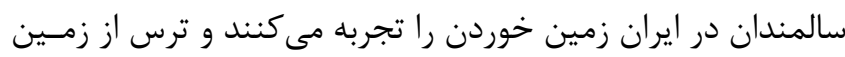

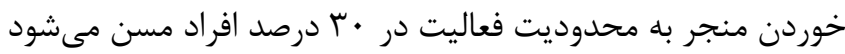

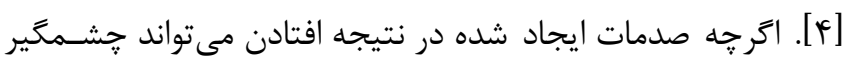

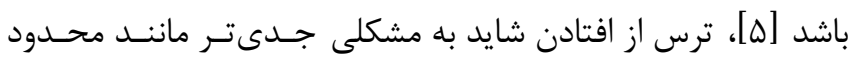

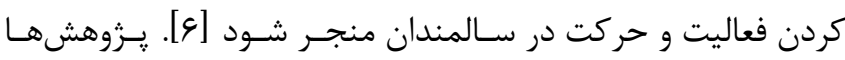

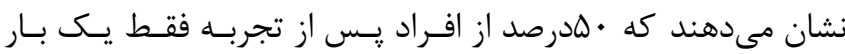

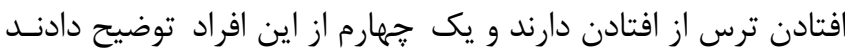

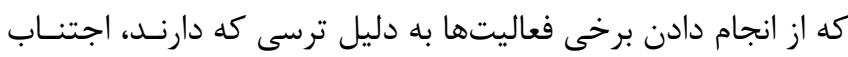

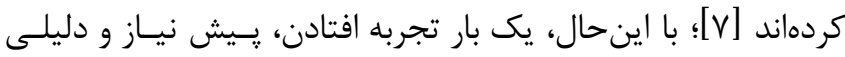

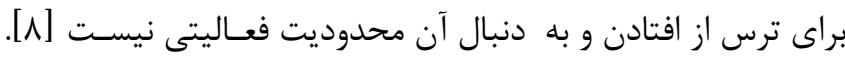

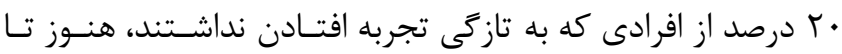

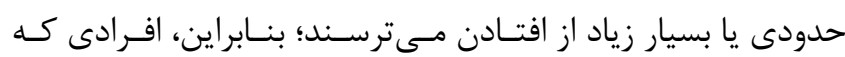

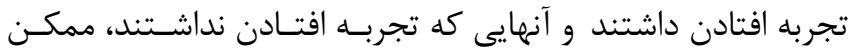

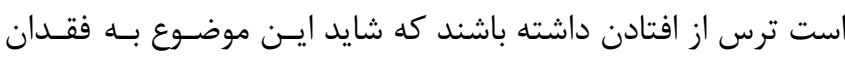

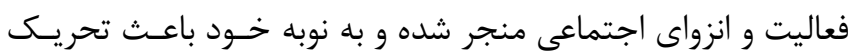
شرطى زدايى، كاهش عملكرد و كـاهش كيفيـت زنــدكى شـود [9].

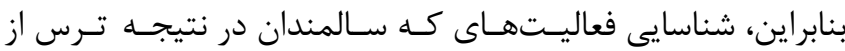

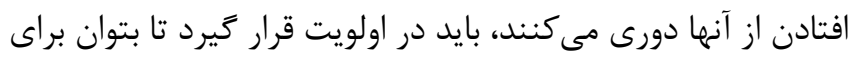

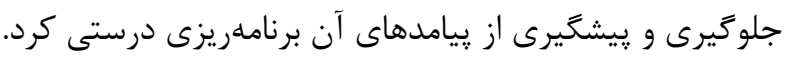

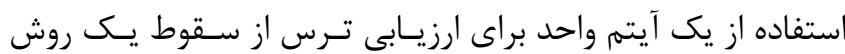

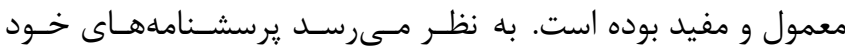

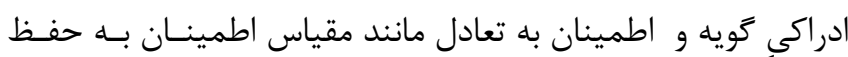

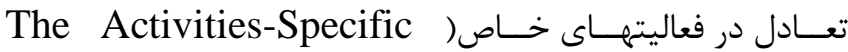
خد (Balance Confidence Scale-ABC خودكسار آمسدى افتـادن(Fefficacy Scale- FES )

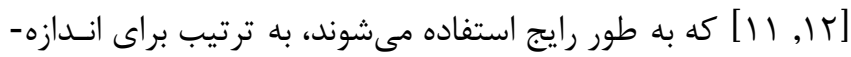
كيرى اعتماد به نفس و خودكار آمدى در فعاليتهاى روزمره زندكى 
اين يرسشنامه در جمعيت سالمندان در ايالات متحده آمريكا مـورد

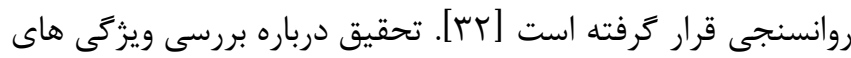

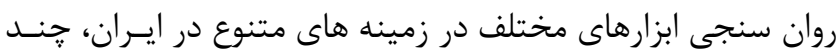

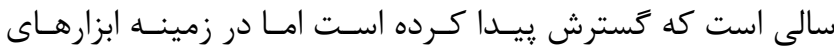
سنجش ترس از افتادن در سالمندان تحقيق ، صورت نكَرفته اسـت؛

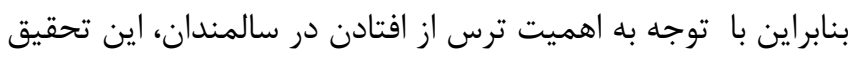

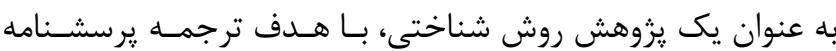

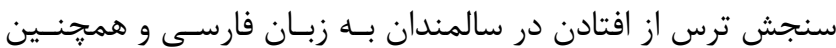

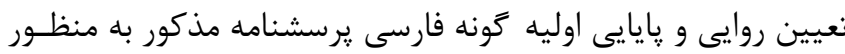

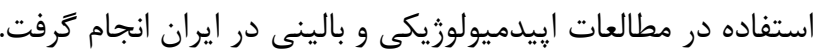

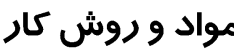

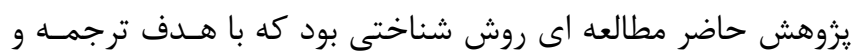

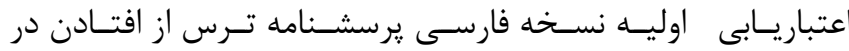

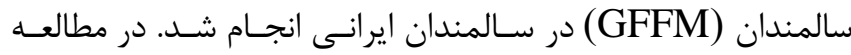

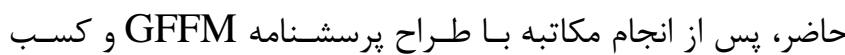

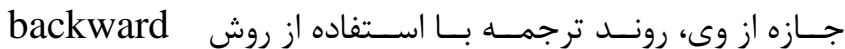
forward

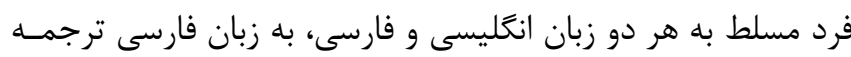
كرديد. سيس دو ترجمه فارسى برسشنامه، توسط تيم بزوهش مون مورد

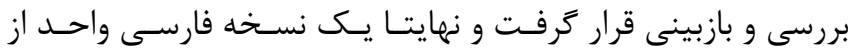

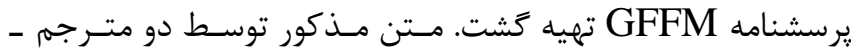

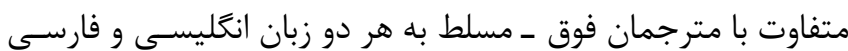

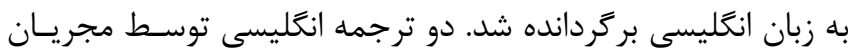

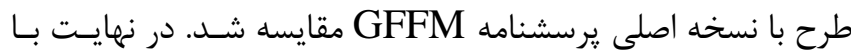

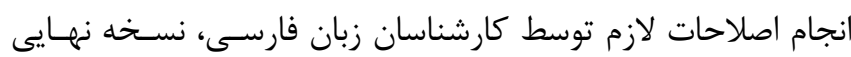

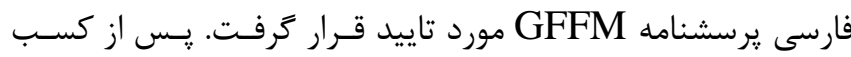
اطمينان از صحت انتقال مفاهيم، به منظور و بررسى روايسى صـورى

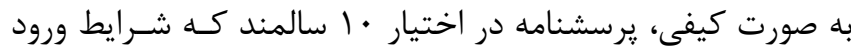

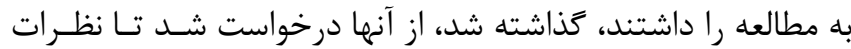

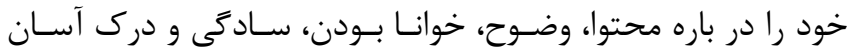
عبارات ابزار و سهولت تكميل يرسشنامه به شكل كيفى بيان كنـــد.

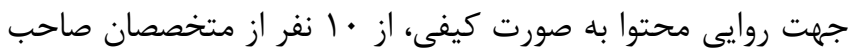

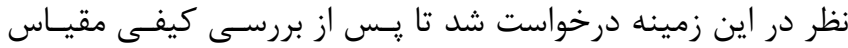

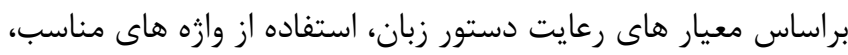

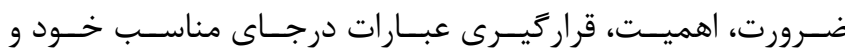

اندازه كَيرى مفيد ترى از ترس از افتادن و آثار آن بر محدود كـردن

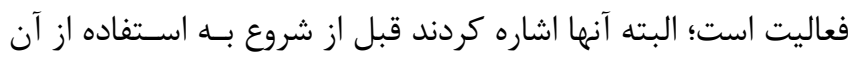

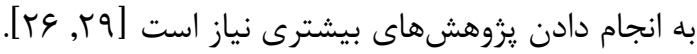

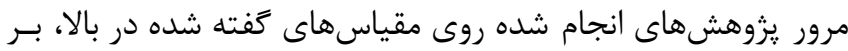

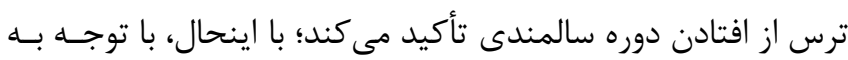

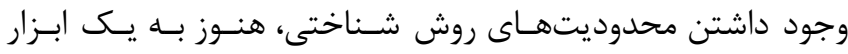

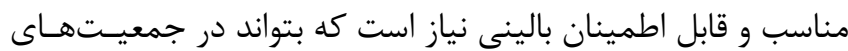
نا همخون مورد استفاده قرار كيرد.

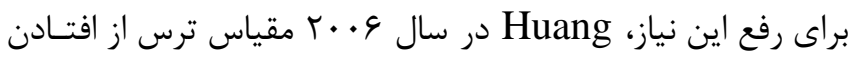

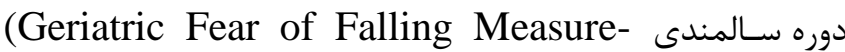
GFFM) براى جمعيت سالمندان در خانسه را سـاخت [•r؟]، ابـزار

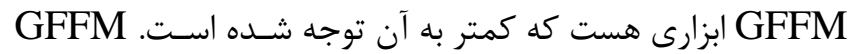

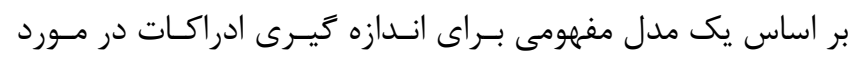
سقوط و شرايط زمانى ترس از سقوط در ميان جامعـهـ-بزر گســالان

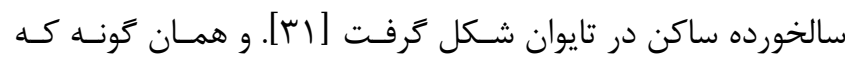

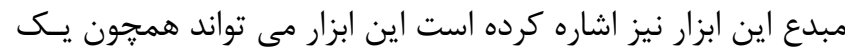

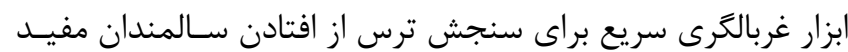

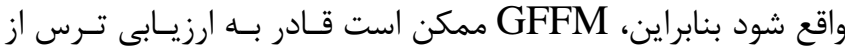

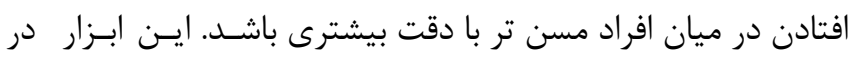

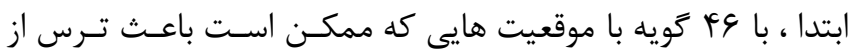
افتادن شود توليد شد و در جهار بعد تقسيم شـد: علائسم روان تنسى إنى

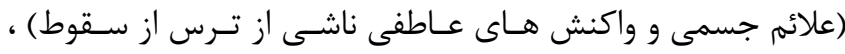

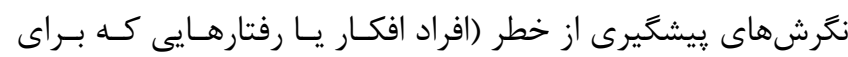
جلو كيرى از سقوط و آمادگى براى شرايط اضـطرارى اسـتفاده مسى إنى

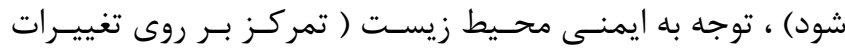

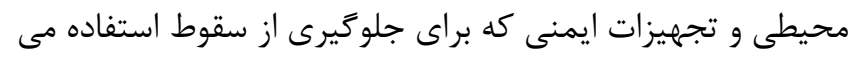

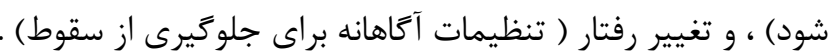

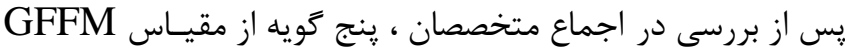

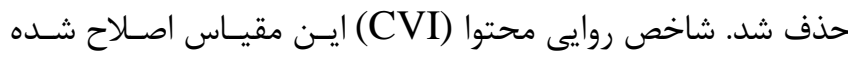

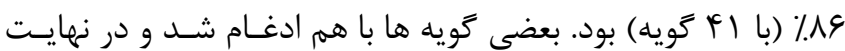

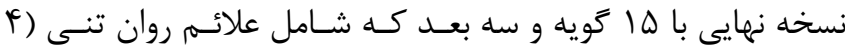

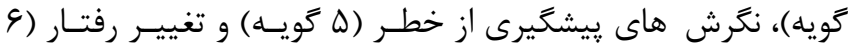

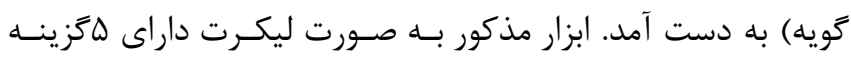

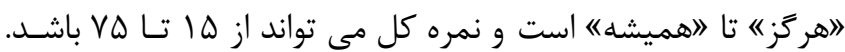
نمره بالاتر نشان دهنده ترس بيشتر از سقوط است. ميـانخين مـدات التهات

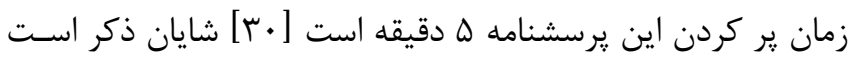


ميـزان تحصـيلات، كيفيـت وضـعيت اقتصـادى، وضـعيت اشـتغال، وضعيت سلامتى، تعداد فرزندان است. در مرحلـه روان ســنجى، بــهـ منظور تعيين روايى سازه و در ياسخ بـه اين سؤال كه آيا گويه هاى

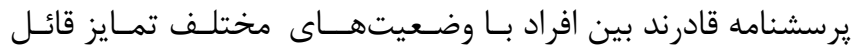
شوند، از روش مقايسه گروههاى شـناخته شـده استفاده شـد. بــراى

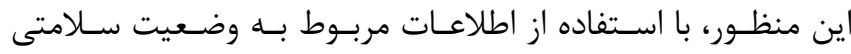

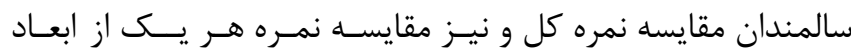

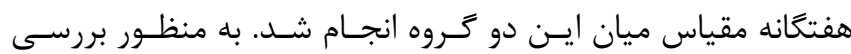

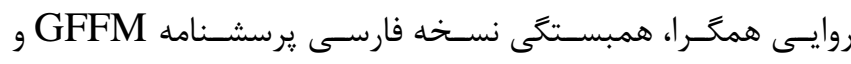

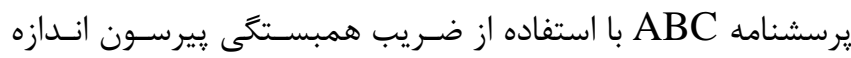
گيرى گرديد. براى تعيين زايايى از شيوه همسـانى درونسى اسـتفاده شد. جهت اندازه كيرى همبسـتكى درونسى ضـريب آلفــى كرونبـاخ

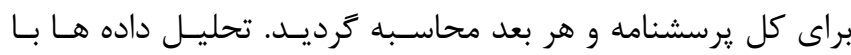
ستفاده از نرم افزار SPSS 24 انجام گرديد.

\section{يافتهها}

در مجموع، • له سالمند مراجعـه كنـــده بـه سـراى محـلات شهمر تهران، به صورت تصادفى طبقه ایى انتخـاب شـدند و يـس از اعـلام

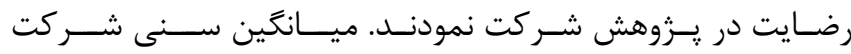

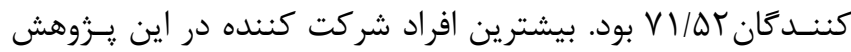

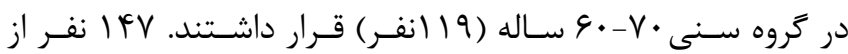
شركت كنند

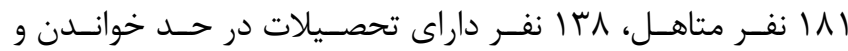

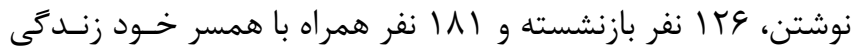

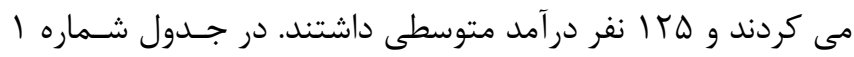
درصد فراوانى ويزگگى هاى جمعيتى مورد مطالعه آمده است. نتايج به دست آمده از تجزيه و تحليل واريانس يك طرئ طرفه نشـان داد ماد كه GFFM به طور قابل توجهى مى تواند در زير گروه هاى بزر

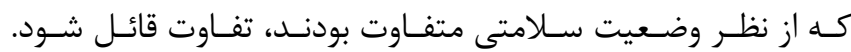
سالمندانى كه از سلامتى متوسطى برخوردار بودند ، بــه طـور قابـل

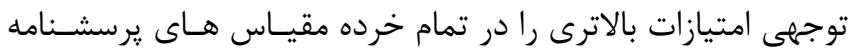

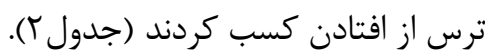

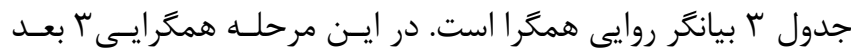

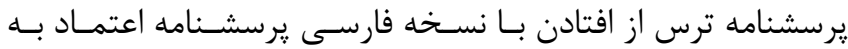

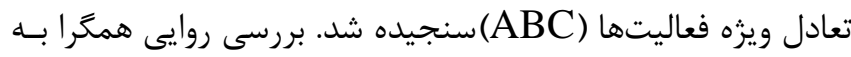

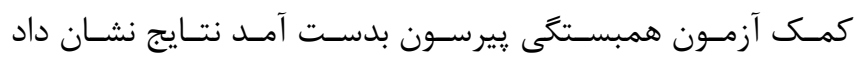

امتيازدهى مناسب، بازخورد لازم را ارائـه دهنــد و در نهايـت نسـخه فارسى يرسشنامه GFFM تهيه شد. جامعه هدف يزوهش سالمندان مراجعه كننده به سراى محلات شهر

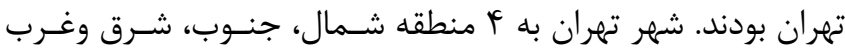

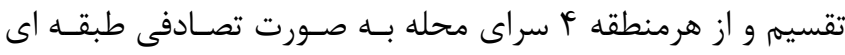
انتخاب شد. نمونه گيرى در هر يك از سراى محله ها نيز به صـورت

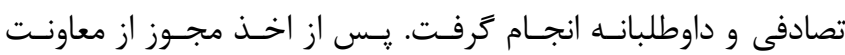
يزوهشى دانشگاه و نيز هماهنكى با سراى محلات و سـالمندانى كـهـ

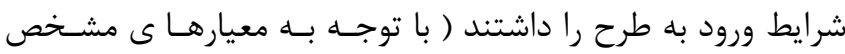

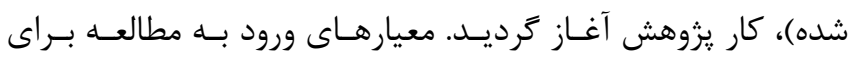
سالمندان عبارت بودند از: سالمندان داراى سن •ع سـال يـا بـالاتر، تمايل سالمندان نسبت به شركت در يـرزوهش، سـكونت در منــاطق

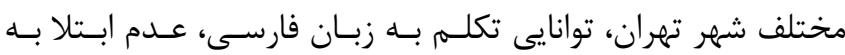

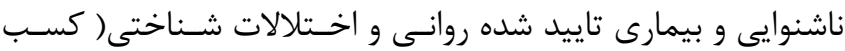

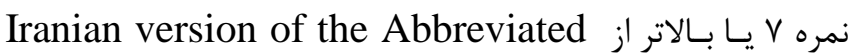
(Mental Test Score

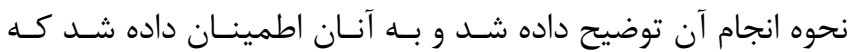
تمامى اطلاعات مربوط به آنها محرمانه نزد يزوهشگران خواهد ماند؛

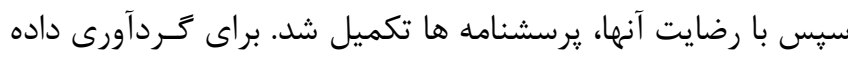

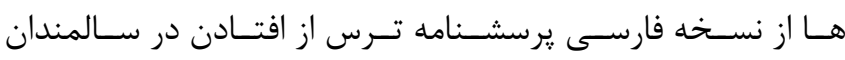

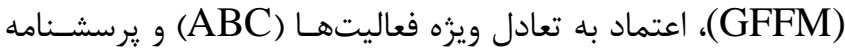
ويزگ

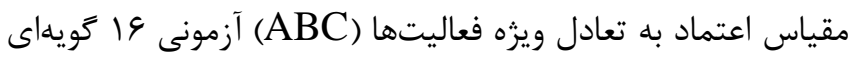
است كه از آزمودنىها مى خواهد اعتماد خود را (.٪ •=عـدم اعتمـاد و

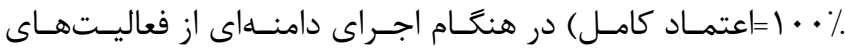

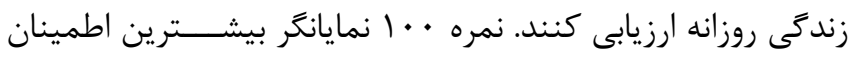

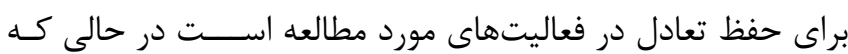
نمره كمتر از • ^ به عنوان شـــشرايط نيازمند مداخله بــراى بهبـود

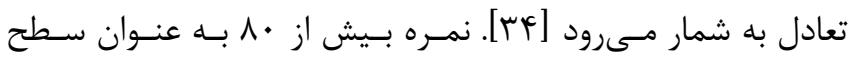

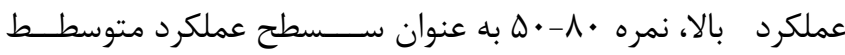
و نمره كمتر از •له به عنوان سـسطح عملكرد يائين در نظر گرفتـه

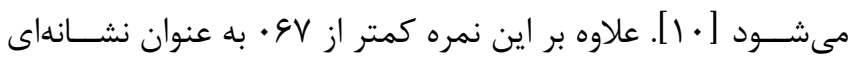

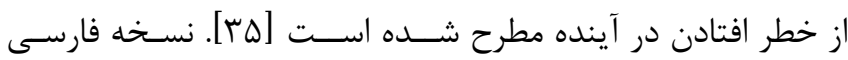

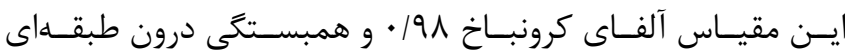
191 ( ICC) شناختى شامل سن، جنسـيت، وضـعيت تاهـل، وضـعيت سـكونت، 


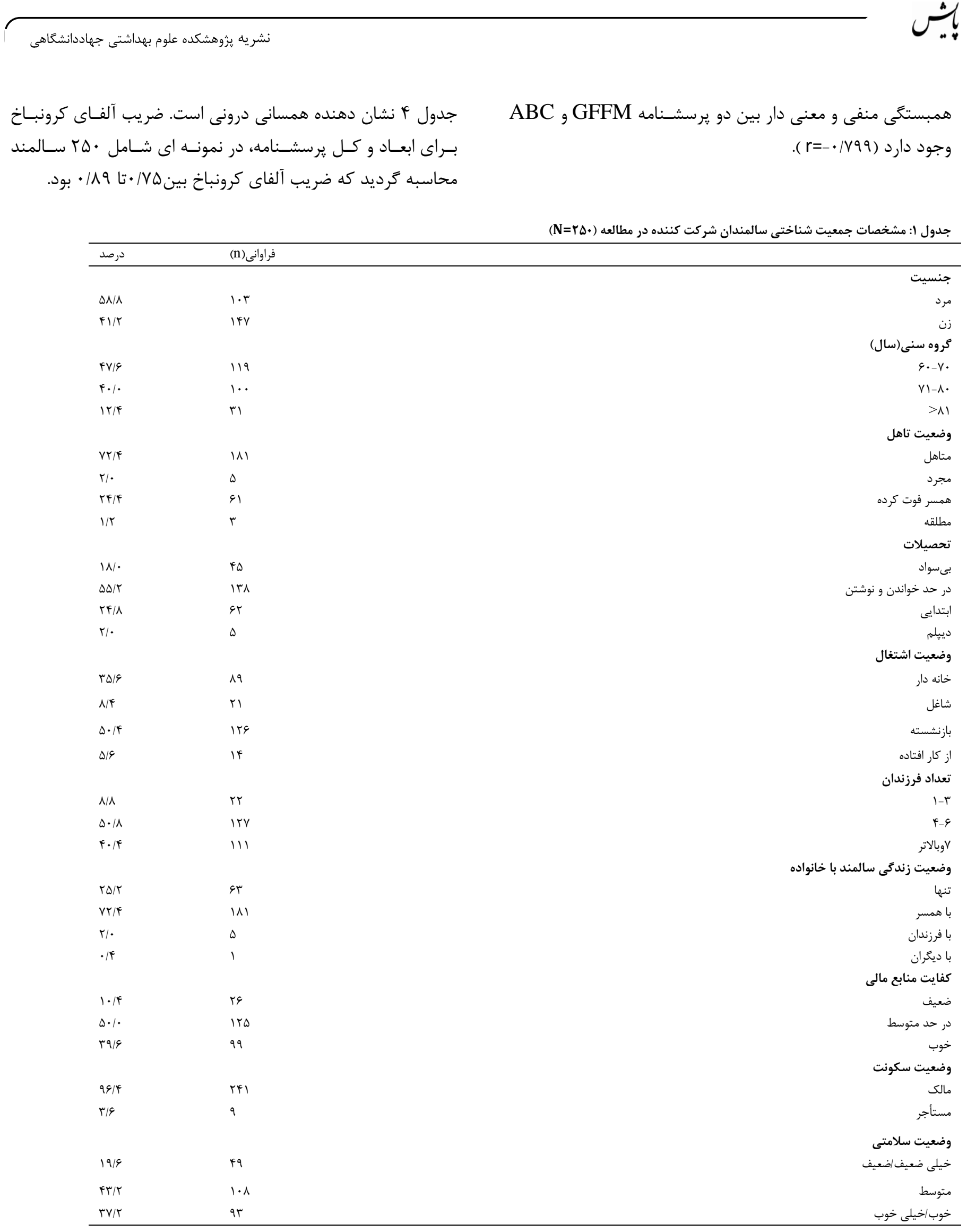


سال بيستم، شماره سوم، خرداد ـ تير ...

جدول r: مقايسه تروه هاى شناخته شده : ميانگين امتياز ابعاد نسخه فارسى ترس از افتادن در سالمندان (GFFM) بر مبناى وضعيت سلامتى

\begin{tabular}{|c|c|c|c|c|}
\hline \multirow[t]{2}{*}{$\mathrm{P}$} & \multicolumn{3}{|c|}{ ميانكَين(انحراف معيار)بر مبناى وضعيت سلامتى } & ابعاد \\
\hline & $\begin{array}{l}\text { خوب/خيلى خوب (n=q) } \\
\text { خو= (1) }\end{array}$ & $\begin{array}{l}\text { متوسط } \\
(n=1 \cdot 1)\end{array}$ & ضعيف/خيلى ضعيف & مقياس \\
\hline$\cdot / \cdot r q$ & $I T / T V(T / A V)$ & $|F /| r(T / q \mid)$ & $|r /| \varepsilon(T / \varepsilon \wedge)$ & علايم روانتنى \\
\hline$\cdot / \cdot r$ & $\mid F / \Delta r(r / T V)$ & $\mid \Delta / T \Lambda(T / 9 \varphi)$ & $\mid r / F \wedge(T / G \psi 4)$ & نغرش حهاى ييشخَيرى از خطر \\
\hline$\cdot 1 \cdot 99$ & १/१९(T/V & $1 \cdot / 1 \cdot(T / 1 T)$ & 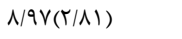 & تغيير رفتار \\
\hline r.|. & $r V / \cdot 1(N / \cdot 1)$ & $r q / \Delta T(\varepsilon / \Delta \Psi)$ & $r \Delta / q \Gamma(V / \cdot \mathcal{\varphi})$ & كل مقياس \\
\hline
\end{tabular}

\begin{tabular}{|c|c|c|c|c|c|}
\hline كل مقياس & تغيير رفتار & نخرشهاى بيشَيرى از خطر & علايم روان تنى & $\begin{array}{l}\text { نمره كل } \\
A B C\end{array}$ & 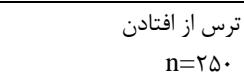 \\
\hline & & . & 1 & 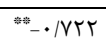 & علايم روان تنى \\
\hline & & 1 & $=0.1949$ & $\stackrel{* *}{*} \cdot / V \cdot 1$ & نكَرشهاى بيشكيرى از خطر \\
\hline & 1 & 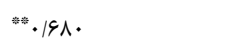 & $* / 09 V$ & $*^{* *} \cdot 1999$ & تغيير رفتار \\
\hline 1 & $\stackrel{* \omega}{* *} \cdot \mid \Lambda \Delta \Lambda$ & $* / 9 \cdot r$ & $\stackrel{m *}{* n} \cdot / \wedge \Delta \Delta$ & $*_{-}^{*} \cdot / \vee 99$ & كل مقياس \\
\hline
\end{tabular}

جدول F: ضرايب آلفاى كرونباخ نسخه فارسى يرسشنامه ترس از افتادن در سالمندان (GFFM)

\begin{tabular}{|c|c|}
\hline$\cdot / V \Delta q$ & علايم روان تنى \\
\hline$\cdot / V \Delta I$ & نغرشههاى بيشگيرى از خطر \\
\hline$\cdot|\Lambda|$ & تغيير رفتار \\
\hline . / 994 & كل مقياس \\
\hline
\end{tabular}

زندَّى آنها را تحت تأثير قرار مىدهد بنابراين بيشـَّيرى از زمسين

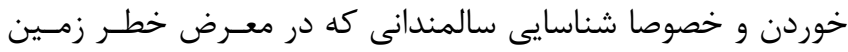

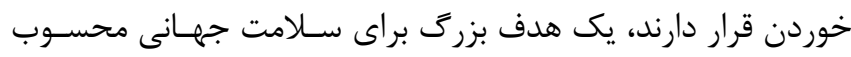

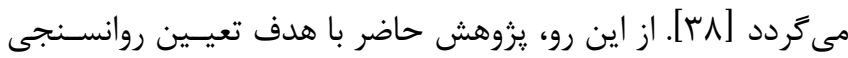

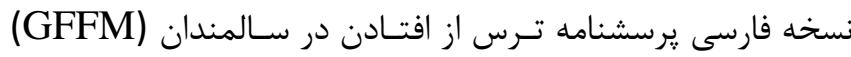

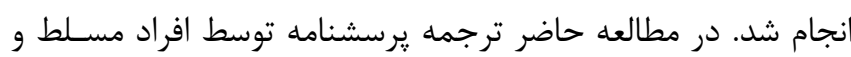

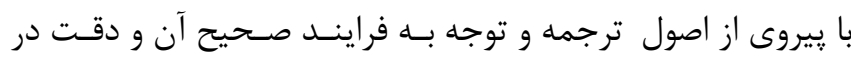

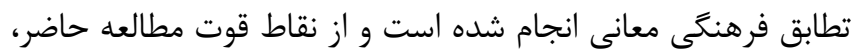

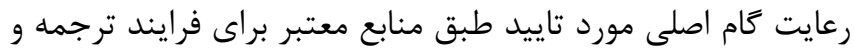

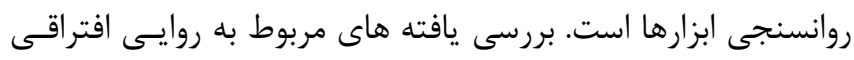

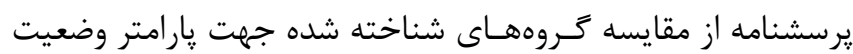

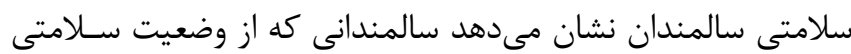

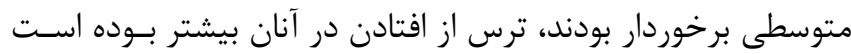

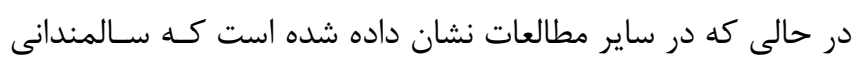

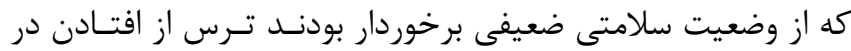

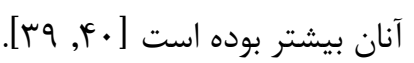

بحث و نتيجه گيرى

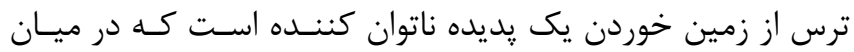

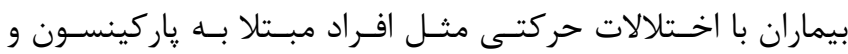

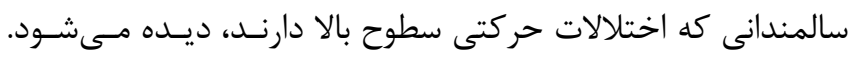

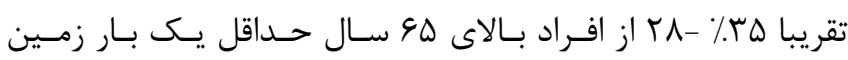

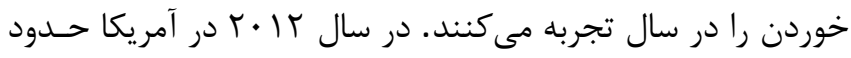

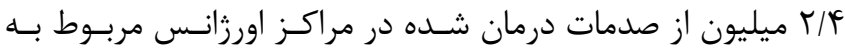

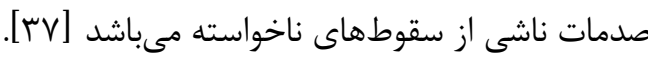

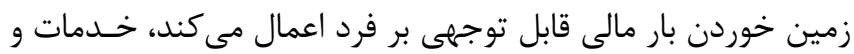

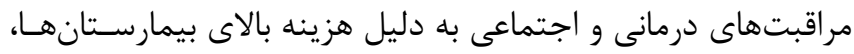

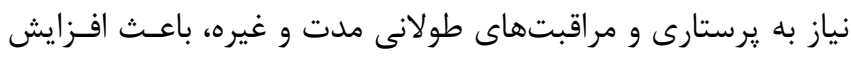

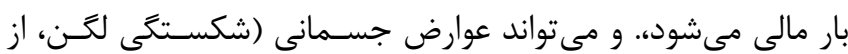

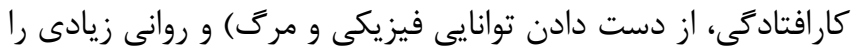

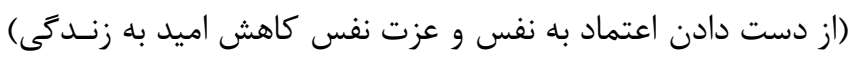

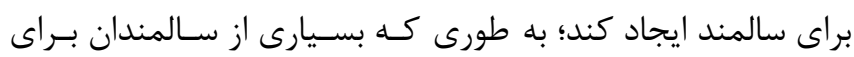

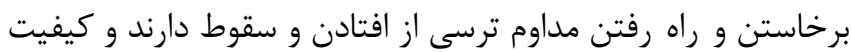




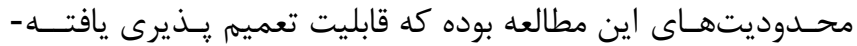

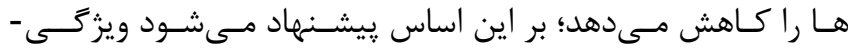

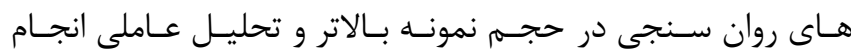

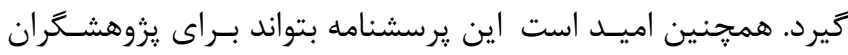
مفيد باشد.

\section{سمم نويسند نان}

نغار صادقى: طراحى طرح نامه، جمع آورى دادهها، ترجمـه و نهـايى كردن يرسشنامه، تهيه و تدوين مقاله

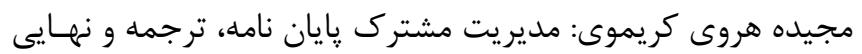

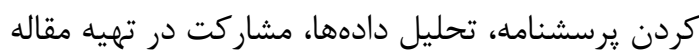

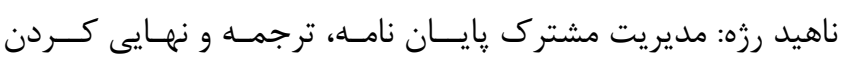
يرسشنامه على منتظرى: مديريت مشترك رايان نامه، ترجمه و نهـايى كـــردن

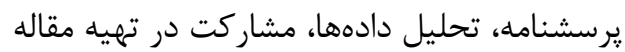

\section{تشكر و قدردانى}

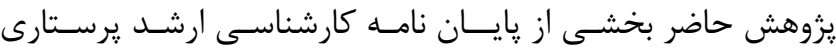

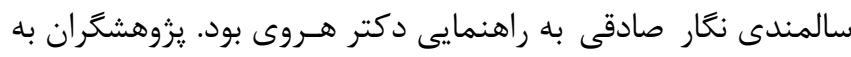

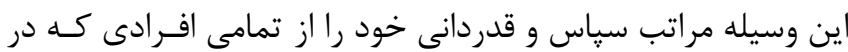

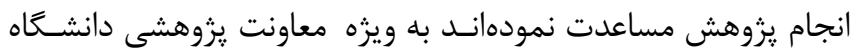
شاهد و سالمندان شـركت كننده در اين يزوهش اعلام مىدارند.

\section{منابع}

1. Domingos C, Correia Santos N, Pêgo JM. Association between Self-Reported and Accelerometer-Based Estimates of Physical Activity in Portuguese Older Adults. Sensors 2021;21:2258

2. Banman D. Decreasing Fall Risk in Older Adults: Understanding Health Care Providers Balance Assessment Practices in Urban and Rural Settings in Saskatchewan. (Doctoral dissertation, University of Saskatchewan) 2021

3. Ambrose AF, Paul G, Hausdorff JM. Risk factors for falls among older adults: a review of the literature. Maturitas 2013;75:51-61

4. Morteza Bagi HR, Ahmadi S, Hosseini M. Demographics of Fall-Related trauma among the Elderly Presenting to Emergency Department; a Cross-Sectional Study. Emergency (Tehran) 2017;5:e8
در يزوهش حاضر، جهت بررسى روايى همخـرا، ضـرايب همبسـتخى

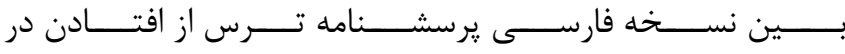

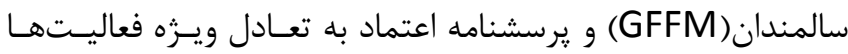
محاسبه كرديد و همبستخى لازم بين نمرات حاصـل از دو يرسشنامه مشاهده شد. در واقع نتايج نشان داد كه هر جــهـ تـرس از

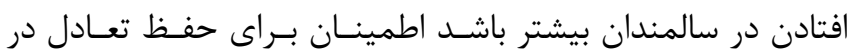

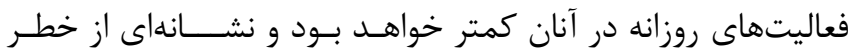
افتادن در آينده براى اين سالمندان مطرح خواهد بود [هـ].

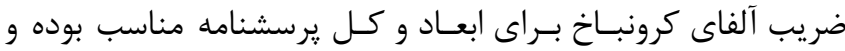

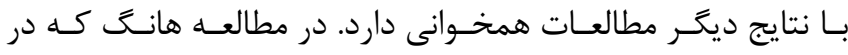

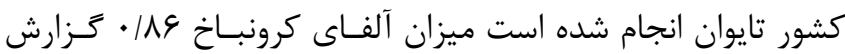

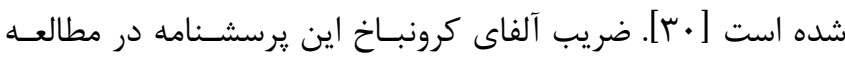

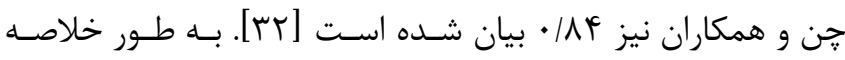

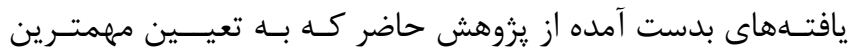
ويزَّى هاى روان ســنجى نسخه فارسى :رسشنامه تـرس از افتـادن در سالمندان يرداخته است، مبين روايى و يايايى يرسشنامه مـذكور

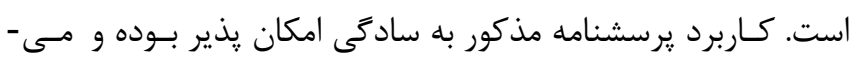

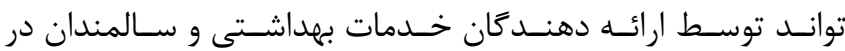
موقعيتهاى مختلـف تكميـل خــردد؛ از ايـن رو اســفاده از ايـنـ از

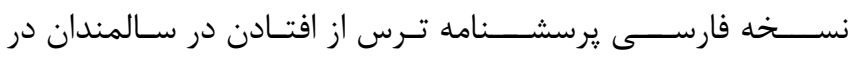

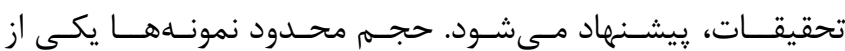

5. Sapmaz M, Mujdeci B. The effect of fear of falling on balance and dual task performance in the elderly. Experimental Gerontology 2021;147:111250

6. Pirrie M, Saini G, Angeles R, Marzanek F, Parascandalo J, Agarwal G. Risk of falls and fear of falling in older adults residing in public housing in Ontario, Canada: findings from a multisite observational study. BMC Geriatrics 2020;20:11

7. Yoshikawa A, Smith ML, Ory MG. Differential risk of falls associated with pain medication among community-dwelling older adults by cognitive status. Age and Ageing 2021 00: 1-8

8. Choi K, Jeon G-S, Cho S-I. Prospective Study on the Impact of Fear of Falling on Functional Decline among Community Dwelling Elderly Women. International journal of environmental Research and Public Health 2017;14:469 
9. Schoene D, Heller C, Aung YN, Sieber CC, Kemmler W, Freiberger E. A systematic review on the influence of fear of falling on quality of life in older people: is there a role for falls? Clinical Interventions in Aging 2019;14:701-19

10. Powell LE, Myers AM. The Activitiesspecific Balance Confidence (ABC) Scale. The journals of gerontology. Series A, Biological Sciences and Medical Sciences 1995;50a:M28-34

11. Tinetti ME, Powell L Fear of falling and low self-efficacy: a cause of dependence in elderly persons. Journal of Gerontology 1993;48:35-38

12. Yardley L, Beyer N, Hauer K, Kempen G, Piot-Ziegler C, Todd C. Development and initial validation of the Falls Efficacy Scale-International (FES-I). Age and Ageing 2005;34:614-9

13. Park S-H. Tools for assessing fall risk in the elderly: a systematic review and meta-analysis. Aging Clinical and Experimental Research 2018;30:1-16

14. Berg K, Wood-Dauphine S, Williams JI, Gayton D. Measuring balance in the elderly: Preliminary development of an instrument. Physiotherapy Canada 1989;41:304-11

15. Bogle Thorbahn LD, Newton RA. Use of the Berg Balance Test to predict falls in elderly persons. Physical Therapy 1996;76:576-83

16. Dibble LE, Lange M. Predicting falls in individuals with Parkinson disease: a reconsideration of clinical balance measures. Journal of Neurologic Physical Therapy 2006;30:60-7

17. McConvey J, Bennett SE. Reliability of the Dynamic Gait Index in individuals with multiple sclerosis. Archives of Physical Medicine and Rehabilitation 2005;86:130-3

18. Chiu Y-P, Fritz SL, Light KE, Velozo CA. Use of item response analysis to investigate measurement properties and clinical validity of data for the dynamic gait index. Physical Therapy 2006;86:778-87

19. Morris S, Morris ME, Iansek R. Reliability of measurements obtained with the Timed "Up \& Go" test in people with Parkinson disease. Physical Therapy $2001 ; 81: 810-8$

20. Duncan PW, Studenski S, Chandler J, Prescott B. Functional reach: predictive validity in a sample of elderly male veterans. Journal of Gerontology 1992;47:93-8

21. Wallmann HW .Comparison of elderly nonfallers and fallers on performance measures of functional reach, sensory organization, and limits of stability. The Journals of Gerontology Series A: Biological Sciences and Medical Sciences 2001;56:580-3

22. Clark S, Rose DJ, Fujimoto K. Generalizability of the limits of stability test in the evaluation of dynamic balance among older adults. Archives of Physical Medicine and Rehabilitation 1997;78:1078-84

23. Deshpande N, Metter EJ, Lauretani F, Bandinelli S, Guralnik J, Ferrucci L. Activity restriction induced by fear of falling and objective and subjective measures of physical function: a prospective cohort study. Journal of the American Geriatrics Society 2008;56:615-20

24. Deshpande N, Metter EJ, Bandinelli S, Lauretani F, Windham BG, Ferrucci L. Psychological, physical and sensory correlates of fear of falling and consequent activity restriction in the elderly: The InCHIANTI Study. American journal of Physical Medicine \& Rehabilitation/Association of Academic Physiatrists 2008;87:354

25. Hotchkiss A, Fisher A, Robertson R, Ruttencutter A, Schuffert J, Barker DB. Convergent and predictive validity of three scales related to falls in the elderly. American Journal of Occupational Therapy 2004;58:100-3

26. Moore DS, Ellis R. Measurement of fallrelated psychological constructs among independentliving older adults: a review of the research literature. Aging and Mental Health 2008;12:684-99

27. Lachman ME, Howland J, Tennstedt S, Jette A, Assmann S, Peterson EW. Fear of falling and activity restriction: the survey of activities and fear of falling in the elderly (SAFE). The Journals of Gerontology Series B: Psychological Sciences and Social Sciences 1998;53:43-50

28. Yardley L, Smith H. A prospective study of the relationship between feared consequences of falling and avoidance of activity in community-living older people. Gerontologist 2002;42:17-23

29. Fabre JM, Ellis R, Kosma M, Wood RH. Falls risk factors and a compendium of falls risk screening instruments. Journal of Geriatric Physical Therapy 2010;33:184-97

30. Huang T-T. Geriatric fear of falling measure: Development and psychometric testing. International Journal of Nursing Studies 2006;43:357-65

31. Huang T-T. Managing fear of falling: Taiwanese elders' perspective. International Journal of Nursing Studies 2005;42:743-50 
32. Chen T-Y, Edwards JD, Janke $\mathrm{M}$. Examination of the psychometric properties of the geriatric fear of falling measure among communitydwelling older adults in the United States. Journal of Applied Gerontology 2019;38:791-804.

33. Foroughan M, Wahlund LO, Jafari Z, Rahgozar M, Farahani IG, Rashedi V. Validity and reliability of Abbreviated Mental Test Score (AMTS) among older Iranian. Psychogeriatrics 2017; 17:460-5 34. Myers AM, Fletcher PC, Myers AH, Sherk W. Discriminative and evaluative properties of the activities-specific balance confidence (ABC) scale. The Journals of Gerontology Series A: Biological Sciences and Medical Sciences 1998;53:M287-M94

35. Lajoie Y, Gallagher SP. Predicting falls within the elderly community: comparison of postural sway, reaction time, the Berg balance scale and the Activities-specific Balance Confidence (ABC) scale for comparing fallers and non-fallers. Archives of gerontology and geriatrics 2004;38:11-26

36. Khajavi D, Farrokhi A, Jaberi-Moghaddam AA, Kazemnejad A. Effect of strength and balance training program on maintaining balance and quality of life in older male adults with fear of fall. Iranian Journal of Ageing 2016;11:270-9[Persian]

37. Houry D, Florence C, Baldwin G, Stevens J, McClure R. The CDC Injury Center's response to the growing public health problem of falls among older adults. American Journal of Lifestyle Medicine 2016;10:74-7

38. Florence CS, Bergen G, Atherly A, Burns E, Stevens J, Drake C. Medical costs of fatal and nonfatal falls in older adults. Journal of the American Geriatrics Society 2018;66:693-8

39. Giné-Garriga M, Guerra M, Unnithan VB. The effect of functional circuit training on selfreported fear of falling and health status in a group of physically frail older individuals: a randomized controlled trial. Aging clinical and experimental research 2013;25:329-36

40. Brouwer B, Musselman K, Culham E. Physical function and health status among seniors with and without a fear of falling. Gerontology 2004;50:135-41 